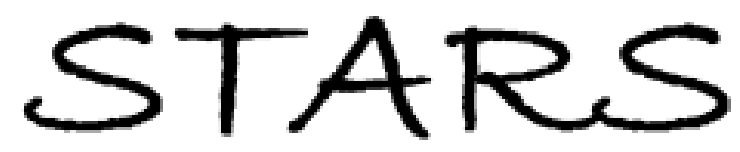

University of Central Florida

STARS

Faculty Bibliography 2000s

Faculty Bibliography

$1-1-2009$

\title{
Electro-optics of polymer-stabilized blue phase liquid crystal displays
}

Zhibing Ge

University of Central Florida

Sebastian Gauza

University of Central Florida

Meizi Jiao

University of Central Florida

Haiqing Xianyu

University of Central Florida

Shin-Tson Wu

University of Central Florida

Find similar works at: https://stars.library.ucf.edu/facultybib2000

University of Central Florida Libraries http://library.ucf.edu

This Article is brought to you for free and open access by the Faculty Bibliography at STARS. It has been accepted for inclusion in Faculty Bibliography 2000s by an authorized administrator of STARS. For more information, please contactSTARS@ucf.edu.

\section{Recommended Citation}

Ge, Zhibing; Gauza, Sebastian; Jiao, Meizi; Xianyu, Haiqing; and Wu, Shin-Tson, "Electro-optics of polymerstabilized blue phase liquid crystal displays" (2009). Faculty Bibliography 2000 s. 1562.

https://stars.library.ucf.edu/facultybib2000/1562

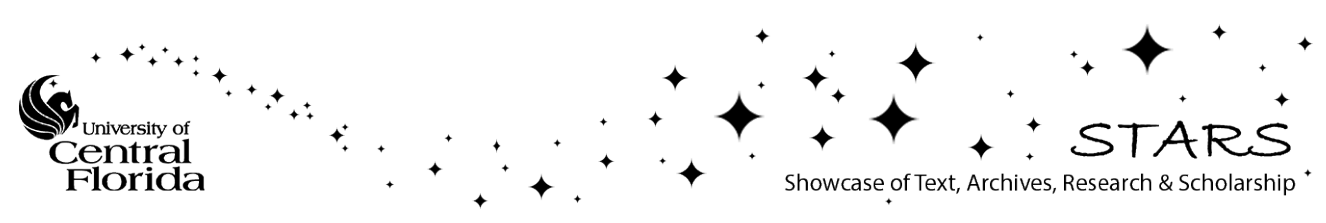




\section{Electro-optics of polymer-stabilized blue phase liquid crystal displays}

Cite as: Appl. Phys. Lett. 94, 101104 (2009); https://doi.org/10.1063/1.3097355

Submitted: 22 January 2009 . Accepted: 19 February 2009 . Published Online: 10 March 2009

Zhibing Ge, Sebastian Gauza, Meizi Jiao, Haiqing Xianyu, and Shin-Tson Wu

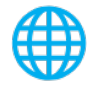

\section{ARTICLES YOU MAY BE INTERESTED IN}

Low voltage blue-phase liquid crystal displays

Applied Physics Letters 95, 231101 (2009); https://doi.org/10.1063/1.3271771

Extended Kerr effect of polymer-stabilized blue-phase liquid crystals

Applied Physics Letters 96, 071105 (2010); https://doi.org/10.1063/1.3318288

A large Kerr constant polymer-stabilized blue phase liquid crystal

Applied Physics Letters 98, 081109 (2011); https://doi.org/10.1063/1.3559614

\section{Applied Physics Letters}

Mid-IR and THz frequency combs special collection

Read Now! 


\title{
Electro-optics of polymer-stabilized blue phase liquid crystal displays
}

\author{
Zhibing Ge, Sebastian Gauza, Meizi Jiao, Haiqing Xianyu, and Shin-Tson Wu ${ }^{\text {a) }}$ \\ College of Optics and Photonics, University of Central Florida, Orlando, Florida 32816, USA
}

(Received 22 January 2009; accepted 19 February 2009; published online 10 March 2009)

\begin{abstract}
Electro-optics of polymer-stabilized blue phase liquid crystal displays (BP LCDs) is analyzed and validated experimentally. A numerical model for characterizing and optimizing the electro-optical and display properties of BP LCDs in in-plane switching and fringe field switching cells is developed. The simulated voltage-dependent transmittance curves agree well with the measured results. To lower the operating voltage while keeping a high transmittance, both electrode width and gap, and large Kerr constant make important contributions. A wide-view BP LCD using a single biaxial compensation film is simulated. (C) 2009 American Institute of Physics.
\end{abstract}

[DOI: $10.1063 / 1.3097355$ ]

Blue phase liquid crystals (BPLCs) are often observed in a very narrow temperature range $\left(\sim 2{ }^{\circ} \mathrm{C}\right)$ between helical cholesteric and isotropic phases. ${ }^{1-3}$ Based on Meiboom's model, ${ }^{2}$ BPLCs are comprised of double twist cylinders arranged in a cubic lattice. The coexistence with disclination lines stabilizes such three-dimensional periodic structures. Recently, the temperature range of BPLCs is expanded significantly by polymerizing a mixture of monomers that could be localized at the disclination cores. ${ }^{4-7}$ The symmetric cubic structures in a polymer-stabilized BPLC appear optically isotropic at zero electric field, but becomes anisotropic when a strong field is applied to induce birefringence. The microscopic structure of such induced birefringence is still not yet completely understood but macroscopically, it can be treated as Kerr effect in isotropic materials caused by the electric field-induced reorientation of polar molecules.

For display applications, polymer-stabilized BPLC offers several advantages including the following: (1) it does not require any alignment layer so that the fabrication process is simple, (2) its voltage-off state is isotropic so that the viewing angle is wide, and (3) its response time is in submillisecond range (depending on the polymer concentration and $\mathrm{BP}$ LC employed) so that it can be used for color sequential displays. Samsung demonstrated a BP LC display (LCD) prototype at 2008 SID exhibition; however, technical detail was not disclosed. Two major challenges need to be overcome before widespread applications can be realized: (1) high operating voltage and (2) low optical efficiency.

In this letter, we develop a numerical model to calculate the induced birefringence of BPLC in fringe-field switching (FFS) (Ref. 8) and in-plane switching (IPS) (Ref. 9) cells. The simulated results agree well with experimental data. Based on these results, we further investigate the electrooptic properties and viewing angle of the biaxial film compensated polymer-stabilized BP LCD.

Figure 1 shows the device configuration of a FFS-BPLC cell (inset) and the measured (dots) and calculated (solid lines) voltage-dependent transmittance (VT) curves. To accurately calculate the detailed molecular distribution of BPLC in response to external fields, both Landau free energy and electric energy need to be considered, ${ }^{1,3}$ which is rather complicated. However, at macroscopic scale the electro-optical

${ }^{a)}$ Electronic mail: swu@mail.ucf.edu. properties can be calculated by a simple model based on Kerr effect. For display applications, a BPLC cell is placed between two crossed linear polarizers and horizontal fringing fields are used to reorient the LC directors, as Fig. 1 shows. Without electric fields, each small blue phase unit can be viewed as an optically isotropic material, having refractive indices identical in its principle coordinates. When a strong electric field $\boldsymbol{E}$ is applied, birefringence is induced and the refractive ellipsoid has its optic axis along the $\boldsymbol{E}$ vector. The induced birefringence $(\Delta n)$ is related to $\boldsymbol{E}$ as ${ }^{10,11}$

$$
\Delta n=\lambda K E^{2}=-n^{3} s_{44} E^{2},
$$

where $\lambda$ is the wavelength, $K$ is the Kerr constant, $n$ is the refractive index of BPLC at its isotropic state, and $s_{44}$ (can be positive or negative) is a quadratic electro-optic effect coefficient. The two following cautions should be taken when interpreting the induced birefringence from Eq. (1): (1) it looks like the induced birefringence is linearly proportional to the wavelength, but actually this is a misconception because Kerr constant is also wavelength dependent and (2) the induced birefringence is linearly proportional to $E^{2}$, but this

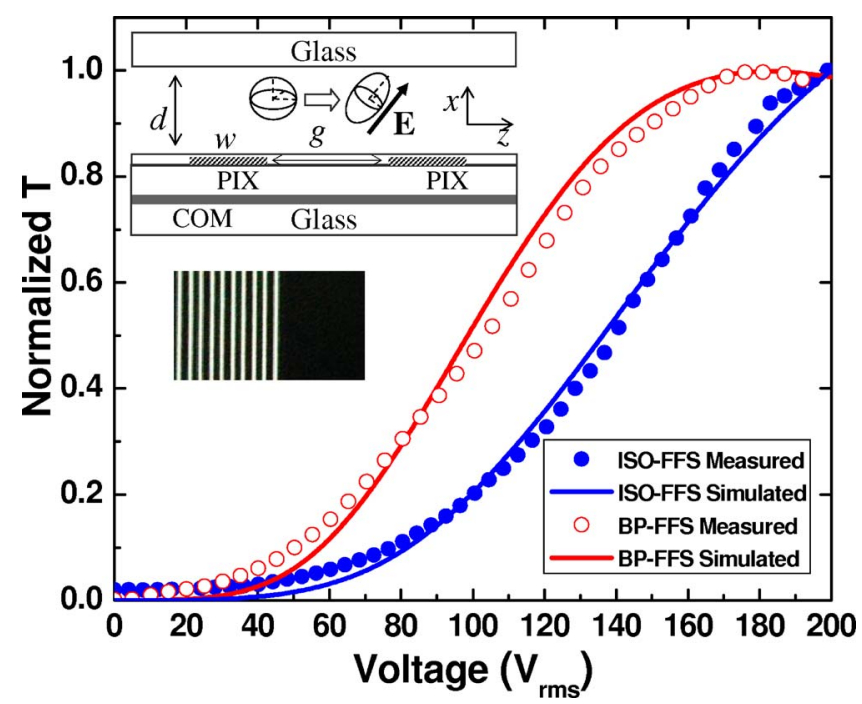

FIG. 1. (Color online) VT curves of FFS cells filled with BPLC sample I (cured at an isotropic phase) and II (cured at a blue phase) at $\lambda$ $=632.8 \mathrm{~nm}$. Cell gap $d=10 \mu \mathrm{m}$, electrode width $w=4 \mu \mathrm{m}$, and electrode gap $g=6 \mu \mathrm{m}$. The photo shows the experimental results of the FFS-BPLC at $90 \mathrm{~V}_{\mathrm{rms}}$. 
is valid only when the electric field is below the saturation electric field $E_{s}$. Otherwise, $\Delta n$ would become unreasonably large when the electric field is very strong. Therefore, it is more informative to rewrite Eq. (1) as

$$
\Delta n=(\Delta n)_{o}\left(E / E_{s}\right)^{2},
$$

where $(\Delta n)_{o}$ stands for the maximum induced birefringence at $E=E_{s}$ and when $E>E_{s}, \Delta n$ saturates. Here $(\Delta n)_{o}$ is related to the LC birefringence, wavelength, and temperature ${ }^{12}$ and $E_{s}$ is governed by the dielectric anisotropy $(\Delta \varepsilon)$, elastic constant, and polymer concentration. A higher birefringence BPLC would lead to a larger induced birefringence, while a larger $\Delta \varepsilon$ and lower polymer concentration (smaller effective elastic constant $)^{13}$ would result in a lower saturation field, but its response time is also slower.

For full color displays, the refractive index dispersion of BPLC should be considered. In Eq. (2), the LC birefringence can be described by the extended Cauchy equation as $(\Delta n)_{o} \approx A^{\prime}+B^{\prime} / \lambda^{2} .{ }^{14}$ As a result, $\lambda K$ has a similar wavelength dependence

$$
\lambda K \approx A+B / \lambda^{2},
$$

where $A$ and $B$ are the modified Cauchy coefficients. The literature data on the wavelength dependent Kerr constant are rare; therefore, we fitted Eq. (3) with the experimental data of $\mathrm{CS}_{2}$ (Ref. 11) and found $A=1.727 \times 10^{-20} \mathrm{~m}^{2} / \mathrm{V}^{2}$ and $B$ $=1.156 \times 10^{-33} \mathrm{~m}^{4} / \mathrm{V}^{2}$. The agreement is quite good (results are not shown here).

In our numerical modeling, we assume the BPLC initially behaves like an isotropic substance. First, we compute the potential distribution from solving the Poisson equation $\nabla(\nabla \cdot \varepsilon \Phi)=0$ and then the distribution of electric field $\boldsymbol{E}$ in the medium. Based on the obtained electric fields, we further calculate the induced birefringence distribution from Eq. (1) and assign the optic axis direction of each unit from the $\boldsymbol{E}$ vector. Considering that the induced birefringence in polymer-stabilized BPLC originates from the reorientation of local LC directors, rather than from the electric field-induced cubic lattice distortion, ${ }^{6}$ we confine the maximum birefringence from Eq. (2) to be below the intrinsic birefringence of the LC system used in the blue phase. After obtaining the birefringence and optic axis profiles, we adopt the extended Jones matrix ${ }^{15}$ to compute the related EO properties.

To validate our simulation, we prepared two FFS cells. One filled with sample I having a host LC material, monomers, and chiral dopants, which was cured in an isotropic state, and the other filled with sample II having the same mixture but cured in the blue phase temperature range. The host LC material used here has a large dielectric anisotropy $(\Delta \varepsilon \sim 32)$ and high birefringence $(\sim 0.30)$. The monomers employed are RM-257 and EHA (ethylhexyl acrylate). LC host material is mixed with monomers at the ratio which enables BP stabilization at room temperature. The FFS cell used in Fig. 1 has a cell gap $d=10 \mu \mathrm{m}$, electrode width $w$ $=4 \mu \mathrm{m}$, and electrode gap $g=6 \mu \mathrm{m}$. In an FFS cell, strong horizontal electric fields exist near the patterned stripe electrode edges; thus high transmittance occurs only near those electrode edges where induced ellipsoid is more horizontally oriented. The photo in Fig. 1 shows an image of the FFS cell filled with sample II, placed between two crossed polarizers, and operated at $90 \mathrm{~V}_{\mathrm{rms}}$. The black area on the right side of the photo represents the dark state.
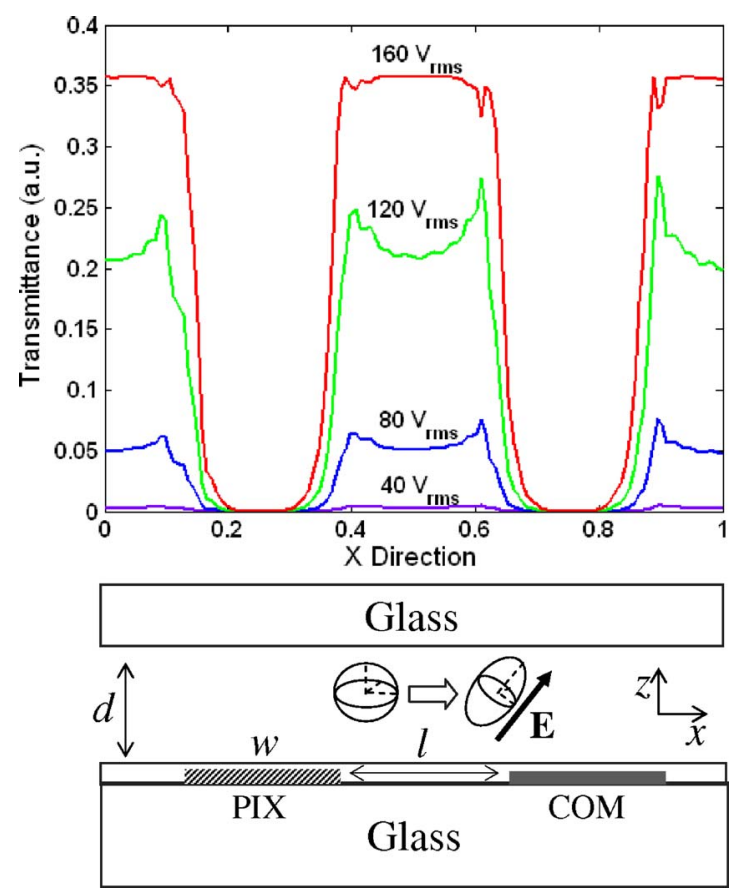

FIG. 2. (Color online) Simulated voltage-dependent transmittance distribution in each period of the IPS cell using BPLC. $\lambda=632.8 \mathrm{~nm}$.

Figure 1 shows the normalized VT curves for FFS cells filled with samples I and II. Dots are experimental data and lines are fitting curves with Kerr constant as the only adjustable parameter. A reasonably good agreement between our simple model and experiment is obtained. Through fittings, we found the Kerr constant for samples I (cured at an isotropic state) and II (cured at blue phase) is $K \sim 4.7$ $\times 10^{-10} \mathrm{~m} / \mathrm{V}^{2}$ and $\sim 1.03 \times 10^{-9} \mathrm{~m} / \mathrm{V}^{2}$, respectively, at $\lambda$ $=632.8 \mathrm{~nm}$. The Kerr constant of sample II is $\sim 2.2$ times larger than that of sample I. For comparison, Kikuchi and co-workers ${ }^{7}$ reported that $K$ can be as large as 3.5 $\times 10^{-9} \mathrm{~m} / \mathrm{V}^{2}$ for their high $\Delta n(\sim 0.199)$ and high $\Delta \varepsilon(\sim 27.6)$ materials. In their measurement, Kerr constants are obtained by directly fitting the measured phase retardation of a BPLC cell in response to applied electric field. Clearly, a larger Kerr constant causes a higher induced birefringence which in turn leads to a lower operating voltage. In our experiment, the measured rise time of the FFS cell using sample II is $\sim 300 \mu$ s at $\sim 23{ }^{\circ} \mathrm{C}$.

Although FFS cell generates strong horizontal fields, they are mainly confined near the electrode edges, resulting in a relatively low transmittance. To enhance transmittance, IPS structure with a larger area having strong horizontal fields is more favorable. Figure 2 depicts the calculated transmittance of an IPS cell with cell gap $d=10 \mu \mathrm{m}$, electrode width $w=10 \mu \mathrm{m}$, and electrode spacing $\ell=10 \mu \mathrm{m}$. Different from the FFS cell shown in Fig. 1, IPS exhibits a high transmittance along the whole area between the pixel and common electrodes. In an IPS cell, besides Kerr constant, the electrode dimension also plays a critical role affecting the maximum transmittance and operation voltage.

Figure 3 depicts the simulated VT curves of different IPS cells at various $w$ and $\ell$ combinations (here IPS: 5-10 denotes $w=5 \mu \mathrm{m}$ and $\ell=10 \mu \mathrm{m})$. For comparison purpose, the results of the FFS cell are also included. The Kerr constant used in the simulations is $1.03 \times 10^{-9} \mathrm{~m} / \mathrm{V}^{2}$ at $\lambda$ $=632.8 \mathrm{~nm}$. As expected, smaller electrode spacing $\ell$ gener- 


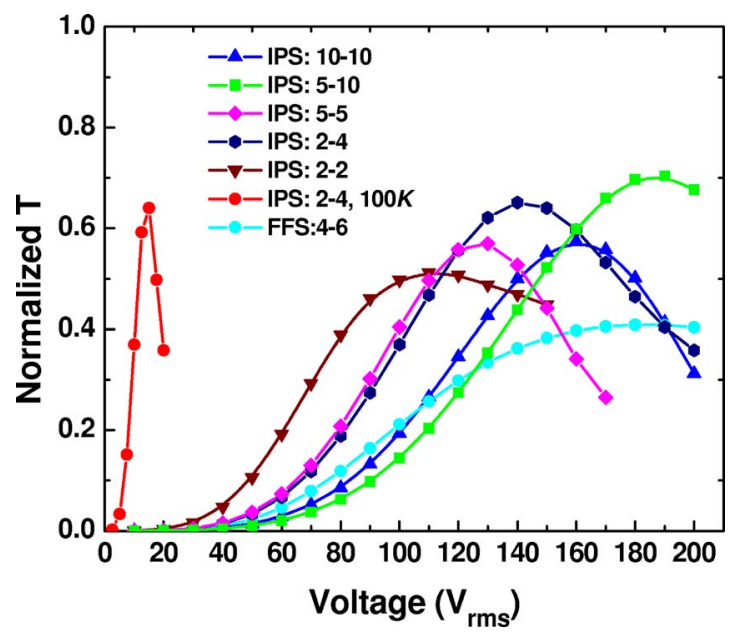

FIG. 3. (Color online) Simulated VT curves of IPS and FFS cells at different electrode dimensions and Kerr constant. $\lambda=632.8 \mathrm{~nm}$.

ally leads to a lower operating voltage. Moreover, a larger $\ell / w$ ratio increases the area ratio having horizontal fields and improves the transmittance. Besides, for a same electrode spacing $\ell$, a larger electrode width $w$ enables the horizontal fields to penetrate deeper into the longitudinal direction (z-direction) which is helpful for lowering the operating voltage. As described before, the FFS cell exhibits a lower transmittance than the IPS one. Particularly, from Fig. 3, the reduction in operating voltage is not proportional to the shrunk electrode width $w$ and spacing $\ell$ [see curves with ( $w$ $=10 \mu \mathrm{m}, \quad \ell=10 \mu \mathrm{m}), \quad(w=5 \mu \mathrm{m}, \quad \ell=5 \mu \mathrm{m})$, and $(w$ $=2 \mu \mathrm{m}, \ell=2 \mu \mathrm{m})]$. Even the electrode width $w$ and spacing $\ell$ decrease to $2 \mu \mathrm{m}$, the on-state voltage remain as high as $100 \mathrm{~V}_{\mathrm{rms}}$. This phenomenon originates from the fact that the dissipation distance of electric fields from the bottom LC cell surface into the longitudinal $z$-direction is roughly proportional to $w+\ell$, which is a common property of Poisson problems (this property is also verified by simulations but results are not shown here). Therefore, although a smaller electrode width $w$ and spacing $\ell$ generates larger electric fields near the surface at a lower voltage, the effective layer having evident induced birefringence is also reduced as compared to that with a larger electrode dimension. As a result, when the electrode dimension shrinks the decrease in operating voltage in IPS cells is balanced by the increased electric field intensity and the thinner effective layer in the longitudinal direction. Although from Eq. (1), induced birefringence is proportional to $E^{2}$, there is certain limit in reducing the driving voltage if electrodes only appear at one side of the substrate. Therefore, to lower the driving voltage, a larger Kerr constant is critically important.

In Fig. 3, the same IPS cell with $w=2 \mu \mathrm{m}$ and $\ell$ $=4 \mu \mathrm{m}$ was calculated by increasing the Kerr constant by 100 times. The operating voltage is reduced by 10 times to $\sim 15 \mathrm{~V}_{\text {rms }}$, which roughly follows the relation of $V_{1} / V_{2}$ $\approx \sqrt{K_{2} / K_{1}}$. Our model, although crude, provides a useful tool to analyze the underlying physics relevant to display applications. Besides electrode structure, a weaker polymer network is also helpful for reducing the driving voltage. A tradeoff is in the slower response time.

Besides high optical efficiency and low operating voltage, wide viewing angle is also very important for display

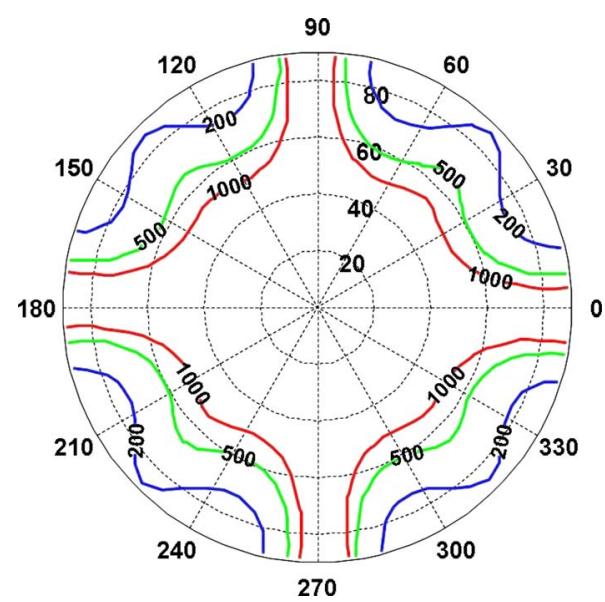

FIG. 4. (Color online) Simulated isocontrast plot of the IPS cell using blue phase LC at $\lambda=632.8 \mathrm{~nm}$ with one biaxial phase compensation film.

applications. Figure 4 depicts the simulated isocontrast plot for the IPS-BPLC cell under two crossed linear polarizers at $\lambda=632.8 \mathrm{~nm}$. The electrode width and spacing are set at $w=10 \mu \mathrm{m}$ and $\ell=10 \mu \mathrm{m}$, and the polarizer has its transmission axis at $\pm 45^{\circ}$ from electrode stripes to warrant a maximum transmittance. As abovementioned, the BPLC behaves like an optically isotropic medium in the voltage-off state; thus the dark state light leakage mainly originates from the effective angle deviation of the two crossed polarizers at oblique angles. ${ }^{16}$ Using a half-wave biaxial plate $\left(n_{x}\right.$ $=1.5110, n_{y}=1.5095$, and $N_{z}=0.5$ ), such light leakages can be dramatically suppressed. In Fig. 4, a contrast ratio larger than 200:1 can be obtained over the $70^{\circ}$ viewing cone.

In conclusion, we have developed simple models to understand the dispersion of Kerr constant for BPLC materials and to predict the electro-optical performance of blue phase LCDs. For an IPS cell, a large Kerr constant and proper electrode width and gap are essential to lower the driving voltage while keeping a high transmittance.

The authors are indebted to the financial support from Chi-Mei Optoelectronics, Taiwan.

${ }^{1}$ P. G. De Gennes and J. Prost, The Physics of Liquid Crystals, 2nd ed. (Clarendon, Oxford, 1993).

${ }^{2}$ S. Meiboom, J. P. Sethna, W. P. Anderson, and W. F. Brinkman, Phys. Rev. Lett. 46, 1216 (1981).

${ }^{3}$ R. Memmer, Liq. Cryst. 27, 533 (2000).

${ }^{4}$ H. Kikuchi, M. Yokota, Y. Hiskado, H. Yang, and T. Kajiyama, Nature Mater. 1, 64 (2002)

${ }^{5}$ Y. Haseba, H. Kikuchi, T. Nagamura, and T. Kajiyama, Adv. Mater. (Weinheim, Ger.) 17, 2311 (2005).

${ }^{6}$ Y. Hisakado, H. Kikuchi, T. Nagamura, and T. Kajiyama, Adv. Mater. (Weinheim, Ger.) 17, 96 (2005).

${ }^{7}$ S. W. Choi, S. I. Yamamoto, Y. Haseba, H. Higuchi, and H. Kikuchi, Appl. Phys. Lett. 92, 043119 (2008).

${ }^{8}$ S. H. Lee, S. L. Lee, and H. Y. Kim, Appl. Phys. Lett. 73, 2881 (1998).

${ }^{9}$ M. Ohe and K. Kondo, Appl. Phys. Lett. 67, 3895 (1995).

${ }^{10}$ J. Kerr, Philos. Mag. 50, 337 (1875).

${ }^{11}$ A. Yariv and P. Yeh, Optical Waves in Crystal: Propagation and Control of Laser Retardation (Wiley, New York, 2002).

${ }^{12}$ S. T. Wu, Phys. Rev. A 33, 1270 (1986).

${ }^{13}$ Y. H. Fan, Y. H. Lin, H. Ren, S. Gauza, and S. T. Wu, Appl. Phys. Lett. 84, 1233 (2004).

${ }^{14}$ J. Li and S. T. Wu, J. Appl. Phys. 95, 896 (2004)

${ }^{15}$ Z. Ge, X. Zhu, T. X. Wu, and S. T. Wu, J. Opt. Soc. Am. A Opt. Image Sci. Vis 22, 966 (2005).

${ }^{16}$ X. Zhu, Z. Ge, and S. T. Wu, J. Disp. Technol. 2, 2 (2006). 